\title{
Embarazo y metabolismo de las proteínas
}

\author{
José Henry Osorio O.*
}

\begin{abstract}
RESUMEN
El papel de las proteínas como bloques edificadores de nuevos tejidos, es fundamental para el crecimiento y desarrollo fetal. La oxidación proteica (sin tener en cuenta el modelo de la fenilalanina), provee la misma cantidad de energía como combustible en todos los estadíos de la mujer embarazada, así como en la que no lo está. Los demás requerimientos extra de energía en el embarazo avanzado son provistos por un incremento en la utilización de los lípidos. En la presente revisión podemos recordar los mecanismos básicos y la secuencia de eventos metabólicos relacionados con las proteínas durante la gestación.
\end{abstract}

PALABRAS CLAVES: Embarazo, metabolismo, proteínas.

\section{SUMMARY}

The role of proteins as the building blocks of new tissue is fundamental to the growth and development of the fetus. Protein oxidation (ignoring the phenylalanine model) provides the same amount of energy for fuel in all stages of pregnancy as in the non-pregnant state. The extra resting energy requirements of late pregnancy are met by increased lipid utilisation. In the present review we can know the basic mechanisms and the metabolic events sequence related to proteins during the pregnancy.

KEY WORDS: Pregnancy, metabolism, proteins.

\section{Introducción}

Los cambios metabólicos durante el embarazo pueden ser considerados como adaptaciones a complejos reajustes fisiológicos los cuales involucran la máxima conservación de energía y el eficiente uso de nutrientes para el mutuo beneficio de la madre y el desarrollo del bebé.

Aunque se han realizado muchos estudios poblacionales y nutricionales sobre investigación proteica o usualmente metabolismo del nitrógeno, debido a implicaciones éticas y técnicas existen pocos estudios que consideren el metabolismo proteico total en el embarazo y en el embarazo de madres diabéticas.

El metabolismo de las proteínas depende de una perfecta coordinación de hormonas maternas y fetoplacentarias, y está relacionado directamente con el metabolismo de carbohidratos y lípidos y el gasto total de energía.

Citaremos el metabolismo general de macromoléculas y el gasto energético para ocupamos finalmente del objetivo fundamental de la presente revisión: el metabolismo de las proteínas.

Profesor Asociado. Director de la línea de Investigación en Bioquímica y Salud. Universidad de Caldas. Manizales. Colombia. Graduate Research Assistant. Child Health Department. Faculty of Medicine. University of Newcastle upon Tyne. England.

\section{Metabolismo de carbohidratos y lípidos durante el embarazo}

En el embarazo normal, las concentraciones de glucosa sanguínea materna son mantenidas a niveles normales o ligeramente subnormales durante el estado de ayuno aunque los niveles postprandiales tienen gran variación (1), y la liberación de insulina en respuesta a la glucosa oral se incrementa en el Embarazo tardío (2). Además en el embarazo avanzado, la producción hepática de glucosa mediante gluconeogénesis se incrementa (3), permaneciendo la glucosa materna y fetal en equilibrio (4), en este estado avanzado, además existe aporte extra de glucosa por incremento en la utilización de los lípidos (5).

Las concentraciones plasmáticas de ácidos grasos no esterificados (NEFA de Non Esterified Fatty Acids) colesterol y triglicéridos se encuentran elevados a través del tercer trimestre (6). Los niveles elevados de triglicéridos pueden ser secundarios a la utilización de NEFA y la reposición de los depósitos grasos depletados (7). Un gradiente de difusión dependiente de pequeñas cantidades de NEFA a través de la placenta ha sido observado, y se estima que el 50\% de los requerimientos fetales de ácidos grasos (en ratas) se derivan de la madre (8).

Un almacenamiento incrementado de lípidos ocurre durante los dos primeros trimestres con una lipólisis similar al de la mujer no embarazada. Almacenamiento al comienzo, como preludio al gasto aumentado al final, es la idea principal. 


\section{Gasto energético durante el embarazo y balance de nitrógeno}

No se puede negar que el crecimiento y desarrollo fetal y placentario, el aumento de tamaño del útero y glándula mamaria, el marcado incremento del volumen sanguíneo y demás cambios en la composición corporal, requieren un incremento extra del gasto energético.

El balance de energía durante el embarazo puede ser influenciado por cambios en la tasa metabólica basal (BMR de Basal Metabolic Rate), la termogénesis y la actividad física.

Se estima que el costo energético total durante el embarazo es de $55.000 \mathrm{Kcal}$. Y los requerimientos totales de energía se han estimado en $2.1,2.3$, y $2.4 \mathrm{Kcal} /$ día, para los tres trimestres sucesivamente (9), aunque existe considerable variación dependiendo de la actividad física (10).

La retención de nitrógeno es una de las necesidades teóricas del embarazo y la ingesta total de energía está correlacionada con la ingestión de nitrógeno, lo cual es similar en no embarazadas (11). El promedio de retención de nitrógeno a las 22 semanas de gestación es similar a las encontradas a término mientras que los valores teóricos basados en análisis químicos de la madre y el feto muestran un incremento progresivo a través del embarazo. La ingestión de nitrógeno además se correlaciona con el balance de nitrógeno, siendo la eficiencia de utilización de nitrógeno entre el 25\%-30\%. El balance de nitrógeno depende entonces de la relación entre síntesis y degradación proteica (12).

La leucina por unidad de peso como una medida de la proteólisis, estuvo reducida en las mujeres embarazadas comparado con no embarazadas, e incrementado en mujeres con diabetes mellitus gestacional (13) esto depende de niveles de insulina, resistencia a la insulina y concentración de esteroides sexuales, mecanismos hasta ahora pobremente entendidos.

Existe adaptación inducida por el embarazo, para conservar las reservas de proteína materna en ayunos cortos (14), a la vez que se presenta correlación linear entre peso fetal y contenido de nitrógeno fetal (15) y una relación exponencial entre la edad gestacional y el contenido de nitrógeno fetal.

Se requiere un incremento diario de proteína promedio, el cual es de $0.64,1.84,4.76$, y 6.1 gramos de las semanas $0-10,10-20,20-30$, y $30-40$ respectivamente para un total de proteína acumulada de 925 gramos y un costo acumulativo de energía total proveniente de la proteína de $21.756 \mathrm{KJ}$ dentro de los $284.596 \mathrm{KJ}$ de energía total neta (16).

Con un peso fetal total de $3.294 \mathrm{~g}$, peso placentario de $644 \mathrm{~g}$ y peso ganado durante el embarazo de $12.500 \mathrm{~g}$, el valor absoluto en gramos de proteína para feto, placenta, líquido amniótico, útero, glándula mamaria, plasma y masa de glóbulos rojos es de 440,100, 3, 166, 81, y 135 respectivamente, para un total de 925 gramos (17).

\section{Cambios metabólico-hormonales durante el embarazo}

El balance entre la tasas de síntesis proteica y la degradación de las mismas determina si existe balance corporal

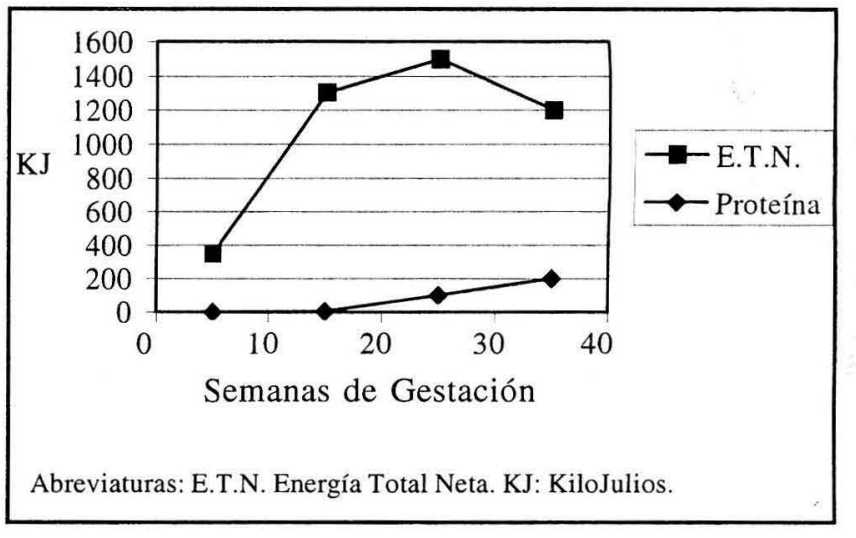

Figura $0016 \mathrm{i01}$

COSTO ACUMULATIVO DE ENERGIA DURANTE EL EMBARAZO

proteico, pérdida o ganancia de la misma. El cambio en estas tasas en respuesta a procesos fisiológicos o patológi$\cos$ es determinado por factores hormonales, insulina, hormona del crecimiento (GH de Growth Hormone), Insulina como factor de crecimiento I IGF-1 factor de insulinoide (IGF-I de Insulin-like Growth Factor I), hormonas tiroideas, esteroides sexuales, glucocorticoides, glucagón y catecolaminas, existiendo estrecha correlación entre sus efectos y no hormonales como estado nutricional, ejercicio, crecimiento e infección (18).

Mientras todas las hormonas mencionadas tienen efectos sobre las tasas de síntesis proteica y proteolisis existe una compleja interacción entre ellas durante el proceso de control. La principal variación tiene que ver con una marcada elevación en la insulina plasmática y mejoramiento de la liberación de insulina postprandial (19). Estos cambios implican un estado de menor sensibilidad a la insulina en tejido muscular y adiposo materno, entendida como una adaptación fisiológica para permitir una dirección preferencial de glucosa y aminoácidos a la unidad feto-placentaria. Se presenta además un incremento progresivo de resistencia a la insulina desde el segundo trimestre llegando a ser marcado en el tercer trimestre (20), la insulina se une al sistema placentario, pero no cruza de la madre al feto, aunque puede existir en circunstancias especiales (21).

El comienzo del embarazo, se caracteriza por incremento de estrógenos y progesterona, lo cual incrementa la proliferación de células B pancreáticas de la madre con una mayor secreción de insulina (22). En etapas avanzadas, la secreción de lactogeno placentario humano (hPL de human Placental Lactogen), la cual tiene un efecto insulinotrópico, se incrementa (23), junto con la prolactina (la cual disminuye la tolerancia a la glucosa) y el cortisol, el cual incrementa la resistencia a la insulina (24). La relación entre estas hormonas es compleja, pero los principales efectos son:

1. Incremento en la respuesta secretora de los islotes (estrógenos, progesterona, hPL) (25).

2. Incremento en la lipólisis vía estrógenos y progesterona y hPL (26). 
3. Resistencia a la insulina (lactógenos placentarios, progesterona, cortisol y otras sustancias no identificadas) (27).

Insulina, GH y GH-I juegan un papel preponderante en la regulación día a día del metabolismo proteico. En humanos, la insulina primariamente inhibe la proteólisis (28) mientras que la GH estimula la síntesis proteica (29). Durante estados post-abortivos IGH-I tiene agudo efecto insulínico sobre la proteolisis, pero durante el estado de alimentación o cuando el sustrato para la síntesis proteica es provisto en forma de infusión de aminoácidos, estimulando la síntesis proteica (30). GH y testosterona tienen un importante papel durante el crecimiento, pero continuan siendo requeridas para mantener la proteína corporal durante el estado adulto (31). Las hormonas tiroideas son requeridas para el normal crecimiento y desarrollo (32). Glucagón, glucocorticoides (33) y adrenalina (34) son todas incrementadas en estados catabólicos y pueden trabajar a la vez en el desdoblamiento de proteína muscular e incremento de la captación de aminoácidos por el hígado para la gluconeogénesis. Mientras un incremento en los glucocorticoides resulta en reducida masa muscular, los efectos del glucagón pueden ser predominantemente hepáticos aumentando la capacidad de captación aminoacídica (35). En contraste al efecto catabólico de la adrenalina sobre el metabolismo de lípidos y carbohidratos, varios estudios sugieren que la adrenalina tiene efectos anticatabólicos en el metabolismo proteico. A pesar de esto, la adrenalina incrementa la producción del aminoácido glucogénico alanina por el músculo y su captación por el lecho esplácnico. Existe un considerable interés en el uso de hormonas anabólicas, solas o en combinación en el tratamiento de estados catabólicos (36). GH combinada con insulina mejora la cinética de músculo esquelético mientras que GH combinada con IGH-I tiene un gran efecto positivo sobre el metabolismo proteico en estados catabólicos que cualquiera de las dos suministradas solas (37).

\section{Función endocrina de la placenta}

Las tres funciones básicas placentarias son:

A. El intercambio gaseoso, comprendido por difusión simple de $\mathrm{O}_{2}$ al feto, promovida por una diferencia de 20 $\mathrm{mmHg}$ de diferencia en la tensión de $\mathrm{O}_{2}$, la alta afinidad por $\mathrm{O}_{2}$ de la hemoglobina fetal y el efecto Bohr, y La transferencia de $\mathrm{CO}$, a la madre por difusión simple (38).

B. La nutrición fetal y la remoción de productos de desecho: transporte de glucosa por difusión facilitada, transporte de grasas por pinocitosis de ácidos grasos libres unidos a albúmina o lipoproteínas, transporte activo de aminoácidos, y síntesis de algunos ácidos grasos (39). La concentración de úrea, ésta es más alta en el feto que en la madre por un margen de cerca de 0.5 $\mathrm{mmol} / 1$ y la tasa de depuración a través de la placenta es de aproximadamente $0.54 \mathrm{mg} / \mathrm{min} / \mathrm{kg}$ de peso fetal a término (40). Se ha sugerido que la proteína provee cerca del $25 \%$ de la fuente de energía para el feto, y que el amonio puede ser transferido a través de la placenta, pudiendo el amonio materno llegar a ser una fuente de nitrógeno fetal (40).
C. Funciones endocrinas y transferencia de inmunoglobulinas (solamente $\operatorname{lgG}$ por pinocitosis): producción de hormonas, incluyendo hCG, progesterona y varios estrógenos (con el feto) (41).

La concentración de aminoácidos libres en el feto es mayor que en la circulación materna (42).

La placenta no toma parte en la síntesis de proteínas fetales aunque sintetiza algunas hormonas proteicas las cuales son transferidas dentro de la circulación materna, principalmente gonadotrofina coriónica y lactógeno placentario humano. La proteína fetal es sintetizada por el feto, de aminoácidos libres transportados a través de la placenta contra un gradiente de concentración. En el embarazo a término, el feto ha acumulado más o menos 500 gramos de proteína (43).

Las Inmunoglobulinas son sintetizadas por el tejido linfoide fetal, y aparece primero la $\lg$ M en la circulación fetal aproximadamente a las 20 semanas de gestación, seguido por la $\lg$ A y finalmente la $\lg$. $\lg$ G es la única gamma globulina que se transfiere a través de la placenta, por pinocitosis, y parece selectiva para el homólogo lgG. No existe evidencia de la transferencia placentaria de hormonas promotoras del crecimiento (44).

\section{Metabolismo proteico en el embarazo normal}

Los nueve aminoácidos esenciales son normalmente tomados de la dieta, los 11 restantes no esenciales tienen varias vías de síntesis a saber:

Alanina puede ser formado mediante una reacción de transaminación utilizando piruvato. Acido aspártico puede ser formado de oxaloacetato por reacción de transaminación. Asparagina por amidación de aspartato. Acido Glutámico, puede ser formado a través de aminación reductiva del alfa cetoglutarato. Glutamina por aminación del glutamato. Arginina mediante el ciclo de la úrea. Prolina en dos etapas provenientes del glutamato. Serina a partir de 3-fosfoclicerato. Glicina, proveniente de serina, mediante la serina hidroximetil transferasa. Cisteina proviene de serina y metionina, siendo entonces no esencial si se suministra metionina en la dieta. $Y$ tirosina formada de fenilalanina, por lo cual es no esencial si se sumistra fenilalanina er la dieta (45).

A las 16-20 semanas de gestación, las concentraciones maternas de aminoácidos son reducidas comparadas con estado de no gravidez, esto incluye la leucina y el aminoácido gluconeogénico alanina (46) y se observan inclusive durante la gestación avanzada, estos cambios ocurren en el embarazo temprano, antes de que ocurra la dilución plasmática y expansión del volumen sanguíneo, una situación similar a la de la glucosa. En el embarazo ha sido encontrado acelerado y potenciado el efecto hipoalaninemico del ayuno (46), ya que la alanina es uno de los mayores precursores gluconeogénicos y se ha sugerido que la hipoalaninemia contribuye a la relativa hipoglicemia del ayuno en el embarazo, consistente con la hipótesis del ayuno acelerado de Freinkel (48), la cual postula que este mecanismo conserva los aminoácidos maternos para la utilización por parte del feto. El peso al nacimiento fetal se correlaciona positivamente con las concentraciones de aminoácidos de la madre para cierto número de aminoácidos (49). 
La síntesis de proteínas es mayor durante el primer trimestre, con niveles bajos durante el tercero. El balance de nitrógeno se hace mas positivo a través del embarazo, en comparación con mujeres no embarazadas y la oxidación proteica no cambia (50). El catabolismo y síntesis proteica en el tercer trimestre son similares en embarazadas y no embarazas, lo que sorprende porque se esperaría un incremento en la demanda metabólica.

$\mathrm{La}$ insulina reduce el catabolismo proteico muscular e incrementa la síntesis proteica (51), para regular la conservación del nitrógeno.

El consenso general sobre el papel de la insulina, es el del balance intra-extracelular de disponibilidad de sustrato para la infusión de aminoácidos y el aporte de flujo proteico para la oxidación (52).

\section{Metabolismo de la proteína fetal}

En trabajos con infusión continua de L-(13C)leucina y $(15 \mathrm{~N})$ fenilalanina en mujeres a término, sometidas a cesárea electiva y mediante análisis de sangre venosa y arterial de cordón umbilical, los principales hallazgos fueron: captación fetal neta de leucina y fenil alanina con flujo de $\mathrm{CO}_{2}$ y ácido cetoisocapróico del feto a la placenta; oxidación fetal de aminoácidos representada en parte por flujo sustancial de la madre; la síntesis de proteína fetal fue más rápida que la observada en adultos, pero similar a la observada en niños pretérmino, con suplantación placentaria de aminoácidos excediendo ligeramente la demanda proteica fetal (53). El feto recibe entonces una adecuada cantidad de aminoácidos de la placenta, mediante incremento en la degradación de proteínas maternas o utilizando los bajos niveles de proteína plasmática de la madre (53).

\section{Embarazo y diabetes mellitus insulino-dependiente (IDDM)}

La deficiencia de insulina es un estado de catabolismo proteico. Estudios in vivo han demostrado que la insulina mejora la captación intracelular de aminoácidos de cadena lateral corta, estimula la transcripción y translación de RNA, incrementa la expresión genética de albúmina y otras proteínas e inhibe el desdoblamiento de enzimas proteicas hepáticas. En pacientes IDDM está incrementada la oxidación de aminoácidos, efecto controlado por la insulina (54).

Un sustancial incremento en la transaminación de leucina durante una deprivación de insulina contribuye al catabolismo de la leucina en estos pacientes. La mayoría de los efectos de la insulina sobre la síntesis proteica parecen ocurrir en tejidos no musculares especialmente en el área esplácnica (55). En adición, la insulina tiene un efecto diferencial sobre la síntesis proteica por el hígado, inhibiendo, por ejemplo, la síntesis de fibrinógeno y promoviendo la síntesis de albúmina, el efecto anticatabólico de la insulina en pacientes IDDM se debe en gran parte entonces, a la inhibición del consumo de proteínas (56). El anabolismo proteico neto debido a la insulina ocurre de manera masiva en músculo esquelético. En pacientes diabéticos no insulino dependientes (NIDDM) estos efectos no son notados presumiblemente por la secreción endógena residual de insulina, por eso, los tratamientos que mejoran el metabolismo de la glucosa en pacientes NIDDM obesos, no afecta el metabolismo proteico (57).

IDDM puede ser considerada como un desorden metabólico caracterizado por hiperglicemia, glicosuria, proteólisis incrementada y cetoacidosis, la cual cede al manejo dietético e insulínico hasta lograr concentraciones de glucosa y lipólisis compatibles con la normalidad (58).

Sin tratamiento, en este tipo de embarazos, puede encontrarse mortalidad materna, fetal, pérdida perinatal (aborto espontáneo, macrosomía fetal, síndrome de estrés respiratorio neonatal) y malformaciones congénitas, además de altos niveles de complicaciones obstétricas al momento del parto (59).

La hipoinsulinemia e hiperglicemia materna facilita el paso de mayor cantidad de glucosa a través de la placenta con hiperglicemia fetal. El páncreas fetal reacciona incrementando la secreción de insulina tratando de normalizar sus niveles de glucosa (60), manteniéndose las condiciones para un rápido y sostenido crecimiento fetal, siendo la macrosomía entonces una complicación asociada con embarazo tardío. Los estudios es este aspecto siguen siendo en algunos casos controversiales, por lo que se aclararan muchos aspectos relacionados en años venideros (61).

Las concentraciones de aminoácidos en madres con diabetes mellitus gestacional son similares a embarazadas normales, sin embargo los aminoácidos de cadena ramificada (valina, leucina, ilsoleucina) se incrementan en diabética gestacionales, comparadas con normales, después de una noche de ayuno (62). Las concentraciones de aminoácidos fueron encontradas elevadas en madres con diabetes mellitus insulino dependiente comparadas con controles y correlacionadas con peso relativo al nacimiento en madres diabéticas (63).

La diabetes mellitus gestacional, usualmente se resuelve en el postparto y tiene implicaciones para el feto y la madre, siendo el síndrome de macrosomía el principal problema clínico donde las calorías son almacenadas excesivamente en el feto, incrementando este su peso $(4000 \mathrm{~g})$ lo cual puede llevar a parto traumático para la madre y el hijo, es mas probable que éstas madres desarrollen diabetes NIDDM en años posteriores (64).

\section{BIBLIOGRAFIA}

1. Phels RL., Metzer BE., Freinkel N. Carbohydrate metabolism in pregnancy XV1l. Am J Obst Gynae 1891; 140: 730-736.

2. Freinkel N. Banting lecture "Of pregnancy" and Progeny". Diabetes 1980; 29:1023-1035.
3. Chiasson FL., Achkar G.C.E., Ducros F., et al. Glucose turnover and gluconeogenesis during pregnancy in normal and type I (insulinin dependent) diabetic women. Diabetologia, 1989; 32: 475A. 
4. Kalhan SC., D' Angelo LJ., Savin SM., et al. Glucose production in pregnant women at term gestation. J Clin lnvest 1979; 63: 388-394.

5. Kalhan SC., Tserng KY., Gilfillan C., et al. Metabolism of urea and glucose in normal and diabetic pregnancy. Metabolism 1982; 31: 824-833.

6. Coustan DR., Berkowitz RL., Hobbins JC. Tigh metabolic control of overt diabetes in pregnancy. Am J Med 1980; 68: 845-852.

7. Elia M., Livesey G. Theory and validity of indirect calorimetry during net lipid synthesis. Am J Clin Nut 1988; 47: 591-607.

8. Coleman RA. Placental metabolism and transport of lipid. Federation Proceed 1986; 45: 2519-2523.

9. Blackburn BW., Calloway DH. Energy expenditure and consumption of mature pregnant and lacting women. J Am Diet Assoc 1976; 69: 29-37.

10. Prentice AM., Goldberg GR., Davies HL., et al. Energy sparing adaptations in human pregnancy assessed by whole body calorimetry. British J Nut 1989; 62: 5-22.

11. Calloway $\mathrm{DH}$., Spector $\mathrm{H}$. Nitrogen balance as related to calorie and protein intake in active young men. Am J Clin Nut 1954; 2: 405.

12. Calloway DH. Nitrogen balance during pregnancy. In: Winnick $M$ Ed. Nutrition and fetal development. 1972; 2: 79-94.

13. Thompson GN., Halliday D. Protein turnover in pregnancy Eur J Clin Nut 1992; 46: 411-417.

14. Matthews DE., Schwars HP., Yang RD., et al. Relationship of plasma leucine and leucine and ketoisocaproate during a $\mathrm{L}-\left[\mathrm{l}^{13} \mathrm{C}\right]$ leucine infusion in man: a method for measuring human intracellular leucine tracer enrichment. Metabolism. 1982; 31(11): 1105-1112.

15. Sparks JW. Human intrauterine growth and nutrient accretio. Seminars in perinatology. $1984 ; 8: 74-83$

16. Hytten FE., Leitch I. The physiology of pregnancy Oxford. Blackwell Scientific Publications 1971; 102.

17. Hytten FE., Chamberlain G. Clinical physiology in obstetrics. Oxford: Blackwell Scientific Publications 1980; 48

18. Wemerman J., Barle H., Hammarquist F. Tissue-specific effects of growth hormone on protein metabolism. Growth hormone and IGF Res. 1988; 8(SB): 111-113.

19. Ross RJM., Wilmore DW. Endocrinology in catabolic illness. Endocrinology and Metabolis 1996; 3(Suppl 1): 115-118.

20. Cousins L. Insulin sensitivity in pregnancy. Diabetes 1991; 40(Supp 2): $39-43$

21. Menon RK., Cohen RM., Sperling MA., et al. Transplacental passage of insuline in pregnant women with insulin dependent diabetes mellitus. New England J Med 1990; 323(3): 309-315.

22. Forsum E., Sadurskis A., Wager J. Resting metabolic rate and body composition of healthy swedish women during pregnancy Am J Clin Nut $1988 ; 47: 942-947$.

23. Parsons JA., Brejle TC., Sorenson RL. Adaptations of islets of Langerhans to pregnancy: Increased islet cell proliferation and insulin secretion correlates with the onset of hPL secretion. Endocrinol 1992; 130: $1459-1466$

24. Hollingsworth DR. Endocrine and metabolic homeostasis in diabetic pregnancy. Clin Perinatol 1983; 10: 593-614.

25. Russell-Jones DL., Umpleby AM., Hennessy TR., et al. Use of a leucine clamp to demostrate thet IGF-I Actively stimulates protein synthesis in normal humans. Am J Physiology 1994; 267: E591E598

26. Mochizuki M., Morikawa H., Ohga Y. Lipolytic action of human chorionic somatotropin. Endocrinol. Jnp. 1975; 22: 123-129.

27. Russell-Jones DL., Weissberger AJ., Bowes SB., et al. The effects of growth hormone on protein metabolism in adult growth hormone deficient patients. Clinical Endocrinology 1993; 38: 427-431.

28. Russell-Jones DL., Rattray M., Wilson VJ., et al. Intraperitoneal insulin is more potent than subcutaneous insulin at restoring hepatic IGF-ImRNA levels in the diabetic rat: a functional role for the portal vascular link. J Molec Endocrinol 1992; 9: 257-263.

29. Mulligan K., Grunfeld C., Hellerstein MK. et al. Anabolic effect of recombinant human growth hormanoe in patients with wasting associated with human immunodeficiency virus infection. J. Clin Endocrin Metab 1993; 77: 956-962.

30. Ross JC., Fennessy PV., Randall BW. et al. Placental transport and fetal utilisation of leucine in a model of fetal growth retardation. Am J Physiol 1996; 270: E491-E503.
31. Urban RJ., Bondenurg YH., Gilikson C. et al. Testosterone administration to elderly men increases skeletal muscle strengh and protein synthesis. Am J Physiol 1995; 269: E820-E826.

32. Tauveron I., Charrier S., Champredon C. et al. Response of leucine metabolism to hyperinsulinemia under amino acid replacement in experimental hyperthyroidism. Am J Physiol 1995; 269: E499E507

33. Bowes SB., Jackson NC., Papachristodouluo D. et al. Effect of corticosterone on protein degradation in isolated rat soleus and extensor digitorum longus muscles. J Endocrinology 1996; 148 : 771-782

34. Fryburg DA., Gelfand RA., Jahn LA., et al. Effects of epinephrine on human muscle glucose and protein metabolism. Am J Physiology 1995; 268: E55-E59.

35. Fryburg DA., Jahn LA., Hill SA. et al. Insulin and insulin-like growth factor I anhance human skeletal muscle protein anabolism during hyperaminoacidemia by different mechanisms. J Clin lnvest 1995; 96: 1722-1729.

36. Southorn BG., Palmer RM/, Garlick PJ. Acute effects of corticosterone on tissue protein synthesis and insulin-sensitivity in rats in vivo. Biochem J 1990; 272: 187-191

37. Umpleby AM., Shojaee-Moradie F., Thomason M. et al. Effects of insulin-like growth factor I (IGF-I), insulin and combined IGF-Iinsulin infusions on protein metabolism in dogs. Eur J Clin Invest 1994; $24: 337-344$.

38. Symonds EM., Symonds IM. Placental and fetal growth and development. In: Essential obstetrics and gynaecology. 3 ed. Churchill Livingstone London 1998; 59-71.

39. Coleman RA. Placental metabolism and transport of lipid. Federation Proceedings. 1986; 45: 2519-2523

40. Chien PFW., Taylor DJ., Watt PW. et al. Protein synthesis in human term placenta, umbilical cord ans uterus. Proc Nut Soc 1990; 49: $183 \mathrm{~A}$.

41. De Swiet M., Chamberlain GVP. Basic science in obstetrics and gynaecology. Churchill Livingstone Edinburgh 1992; 40-62.

42. Thorbum GD., Harding R. Textbook of fetal physiology. Oxford University Press. Oxford. 1994; 120-130.

43. Dicke JM., Henderson Gl. Placenta amino acid uptake in normal and complicated pregnancies. Am J Med Sci 1988; 295(3): 223-227.

44. Eaton BM., Yudilevich DL. Uptake and asymmetric efflux of amino acids at maternal and fetal sides of placenta. Am J Physiol 1981; 241(3): C106-C112.

45. Davidson V. Biosynthesis of amino acids and amino acids compounds. In: Biochemistry. $3^{\text {rd }}$ edition. The national medical series for independent study. Harwal 1994; chapter 29: 471

46. Felig P., Kim YJ., Lynch V. et al. Amino acid metabolism during starvation in humam pregnancy. J Clin lnvest 1972; 51: 1195-1202.

47. Kalhan SC., Gilfillan CA., T semg KY. Glucose alanine relationships in normal human pregnancy. Metabolism 1988; 37: 152-158.

48. Evain-Brion D. Maternal endocrine adaptations to placental hormones in humans. Acta Paediatrica. 1999; 88(S428): 12-16.

49. Holzman IR., Lemons JA., Meschia G. et al. Uterine uptake of amino acids and placental glutamine-glutamate balance in the pregnant ewe. J Developmental Physiol 1979; 1(2): 137-149.

50. Fitch W., King JC. Protein turnover and 3-methylhistidine excretion in non-pregnant, pregnant and gestational diabetic women. Human nut.: Clin Nut 1987; 41C: 327-339.

51. Fryburg DA., Barret EJ., Louard RJ. et al. Effect of starvation on human muscle protein metabolism and its response to insulin. Am J Physiol 1990; 259: E477-E482.

52. Ang BCN., Halliday D., Wade AJ. et al. Insuline reduces leucine oxidation and improves nitrogen balance in the parenterally-fed state. Proceedings Nut Soc 1992; 51: 111A.

53. Chien PFW., Smith K., Watt PW. et al. Protein tumover in the human fetus studied at term using stable-isotope tracer amino-acids. Am J Physiol 1(1): E31-E35

54. Maresh M., Beard RW., Bray CS. et al. Factors predisposing to and outcome of gestacional diabetes. Obstetrics and Gynaecology 1989; 74: 342-346.

55. Mathews DE., Schwars HP., Yang RD., et al. Relationship of plasma leucine and leucine and ketoisocaproate during a L- $\left[1-{ }^{13} \mathrm{C}\right]$ leucine infusion in man: a method for measuring human intracellular leucine traxcer enrichment. Metabolis. 1982; 31(11) $1105-1112$ 
56. Metzger BE., Phelps RL., Frienkel N. et al. Effects of gestational diabetes on diurnal profiles of plasma glucose, lipids, and individual amino acids. Diabetes Care 1980; 3: 402-409.

57. Denne SC., Patel D., Kalhan SC. Leucine Kinetics and fuel utilisation during a brief fast in human pregnancy. Metabolism. 1991; 40(12): 1249-1256.

58. Dornhorst A., Nicholls JS., Johnston DG. Diabetes and diet in pregnancy. Bailleres Clinical Endocrinology and Metabolism 1990; 4(2): 291-311.

59. Brundenell M., Doddrige M. In: Diabetic pregnancy. Churchill Livingstone London 1989; 90-97.

60. Hellerstrom C., Swenne I. Functional maduration and proliferation of fetal pancreatic B-cells. Diabetes. 1992; 40(Supp 2): 89-93.
61. Lariviere F., Kuprancycz DB., Chiasson JL. et al. Plasma leucine kinetics and urinary nitrogen excretion in intensively trested diabetes mellitus. Am J Physiol 1992; 263: E173-E179.

62. Metzger BE., Unger RH., Frienkel N. Carbohydrate metabolism in pregnancy. XIV. Relationships between circulating glucagon, insulinan, glucose and amino acids in response to a "mixed meal" in late pregnancy. Metabolism 1977; 26: 151-156.

63. Kalkhoff RK., Kandaraki E., Morrow PG. et al. Relationship between neonatal birth weight and maternal plasma amino acid profiles in lean and obese nondiabetic women and type I diabetic pregnant women. Metabolism 1988; 37: 234-239.

64. Cooper BG., Walker M., Daley SE, et al. Whole body protein metabolism in late pregnancy and the effects of insulin. 